\title{
Epitope mapping of antibodies induced with a conserved rhinovirus protein generating protective anti-rhinovirus immunity
}

Janakan Sam Narean ${ }^{1,2}$, Nicholas Glanville ${ }^{2,4}$, Christine M Nunn ${ }^{1}$, Katarzyna Niespodziana ${ }^{3}$, Rudolf Valenta ${ }^{3}$, Sebastian L Johnston ${ }^{2}$, and Gary R McLean ${ }^{1,2,5}$

${ }^{1}$ Cellular and Molecular Immunology Research Centre, School of Human Sciences, London Metropolitan University, London UK

2 Airway Disease Section, National Heart and Lung Institute, Imperial College London, London UK

${ }^{3}$ Division of Immunopathology, Department of Pathophysiology and Allergy Research, Center for Pathophysiology, Infectiology and Immunology, Medical University of Vienna, Vienna, Austria

${ }^{4}$ Present address: VirTus Respiratory Research, London UK

${ }^{5}$ Correspondence to: g.mclean@1ondonmet.ac.uk 


\begin{abstract}
Human rhinovirus (RV) infections are the principle cause of common colds and precipitate asthma and chronic obstructive pulmonary disease (COPD) exacerbations. Currently there is no vaccine for $\mathrm{RV}$ which is largely due to the existence of $\sim 160$ serotypes/strains. We demonstrated previously that immunising mice with highly conserved VP4 and VP2 regions of the RV polyprotein (RV-A16 VP0) generated cross-reactive immunity to RV in vivo. The current study investigated and mapped the epitopes of RV-A16 VP0 that are targets for antibodies in serum samples from VP0 immunisation and RV challenge studies in mice. Recombinant capsid proteins, peptide pools and individual peptides spanning the immunogen sequence (RV-A16 VP0) were assessed for IgG binding sites to identify epitopes. We found that peptide pools covering the C-terminus of VP4, the N-terminus of VP2 and the neutralising NIm-II site within VP2 were bound by serum IgG from immunised mice. The NIm-II site peptide pool blocked IgG binding to the immunogen RV-A16 VP0 and individual peptides within the pool binding IgG were further mapped. Thus, we have identified immunodominant epitopes of RV vaccine candidate RV-A16 VP0, noting that strong IgG binding antibodies were observed that target a key neutralising epitope that is highly variable amongst RV serotypes.
\end{abstract}

Key words: rhinovirus, subunit vaccine, antibodies, epitope, neutralisation 